\title{
Ultrasensitive refractive index sensor based on graphene coated hollow core fiber
}

\author{
Meng Huang ${ }^{1}$, Bing Sun ${ }^{1}$, Wei Wei ${ }^{1}$, Hongdan Wan ${ }^{1}$, Zuxing Zhang ${ }^{1}$ and Lin Zhang ${ }^{1,2}$ \\ 1Advanced Photonic Technology Lab, School of Optoelectronic Engineering, Nanjing University of Posts and \\ Telecommunications, Nanjing 210023, China \\ 2Aston Institute of Photonic Technologies, Aston University, Birmingham B4 7ET, UK \\ b.sun@njupt.edu.cn
}

\begin{abstract}
A high-quality nanolayer graphene (NLG) coated hollow core fiber (HCF) platform has been demonstrated for accurate monitoring of refractive index (RI) changes so far mainly operate in liquids but not in air. The NLG with high index is deposited on the outer surface of the $\mathrm{HCF}$, and an enhanced anti-resonant reflecting guidance is formed, which induces sharp periodic lossy dips in the transmission spectrum. A cute experiment conducted interrogating the transmission intensity of the lossy dip demonstrates a high resolution of $2.73 \times 10^{-6}$ RIU and $a$ sensitivity of $-365.9 \mathrm{~dB} / \mathrm{RIU}$, which is two or three times higher than that of intensity-modulated RI sensors reported previously. We believe that this configuration opens research directions for highly sensitive sensing in researches of chemistry, medicine, and biology.
\end{abstract}

Keywords: hollow core fiber, graphene, refractive index sensing, air

\section{INTRODUCTION}

Fiber-optic refractive index (RI) sensors with advantages of high sensitivity, low cost and compact size, have been employed as gaseous RI measurement. In recent years, surface plasma resonance RI sensors [1], grating-based RI sensors [2-4], and interferometric RI sensors [5-8], have attracted considerable interest. Graphene has attracted a great deal of attention on its fundamental physics and electronic properties due to its unique optical properties, e.g. self-polarization effect, large optical absorption, and relative high nonlinearity [9]. Therefore, many novel graphene based optical sensors have been presented through the combination of graphene and optical fiber, such as reduced graphene oxide based fiber-optic humidity sensor [10], graphene-based interferometer for chemical gas sensing [11], and graphene-coated microfiber for high-sensitivity gas sensing [12].

In this paper, we proposed and experimentally demonstrated a high-quality nanolayer graphene (NLG) coated a HCF based on the anti-resonant reflecting optical waveguide (ARROW), which could be used to measure simultaneously surrounding temperature and the RI of air. A cute experiment conducted interrogating the transmission intensity of the lossy dip demonstrates a high resolution of $2.73 \times 10^{-6}$ refractive index unit (RIU) and a sensitivity of $-365.9 \mathrm{~dB} / \mathrm{RIU}$, which is two or three times higher than that of intensity-modulated RI sensors reported previously.

\section{EXPERIMENT RESULTS}

The schematic construction of the hollow core fiber (HCF) without coating graphene is shown in Fig. 1, which mainly consists of a hollow core (Polymicro Technologies, TSP025150) with an inner diameter of 20 $\mu \mathrm{m}$ and a ring-cladding with a thickness of $53 \mu \mathrm{m}$, the optical image for the cross section of which is illustrated in the inset. Then the graphene was coated on the outer surface of HCF by using Plasma Enhanced Chemical Vapor Deposition (PECVD).

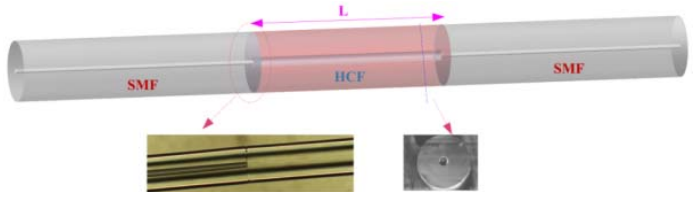

Fig.1 The schematic construction of the HCF without coating graphene.

Prior to the NLG coated the HCF, the transmission spectra are not particularly uniform but appear to include other periodic components. We are inclined to explain that the existed NLG contributes to exciting the dominating anti-resonant reflecting mechanism while suppressing the other higher order anti-resonant reflecting effects. On the other hand, the NLG fabricated by PECVD possesses unique properties compared the reduced graphene oxide provided in this reference [10]. More interesting, the spectra seem to more uniform with more layers of graphene.
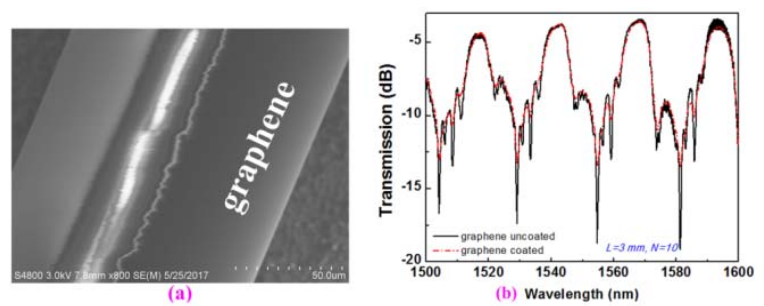

Fig.2. (a) SEM of NLG coated HCF; (b) Transmission spectra of the HCF before and after graphene coating. 
The gas pressure responses of the proposed sensors were tested by means of fixing it into a pressure chamber, where a commercial gas pressure generator (ZC-YFT-02Q) with a stability of $\pm 0.2 \mathrm{kPa}$ and a high precision digital pressure meter were employed to regulate the pressure in the chamber. The applied gas pressure in the chamber was increased from $0.1 \mathrm{MPa}$ to $1.8 \mathrm{MPa}$ at room temperature, and the pressure was maintained for $5 \mathrm{~min}$ at each step. When the pressure increased from $0.1 \mathrm{Mpa}$ to $1.8 \mathrm{Mpa}$, the ambient air RI changed from approximately 1.00026 to 1.00461 . Fig. 3 displays the corresponding evolutions of the transmission spectra and amplitude change as a function of the surrounding refractive index. A linear regression of the raw data yields a sensitivity of $369.5 \mathrm{~dB} / \mathrm{RIU}$, which is two or three times larger than those $(27 \mathrm{~dB} / \mathrm{RIU}$ [13], $94.58 \mathrm{~dB} / \mathrm{RIU}$ [14], and $-110 \mathrm{~dB} / \mathrm{RIU}$ [15]) of the intensity-modulated RI sensors reported previously.

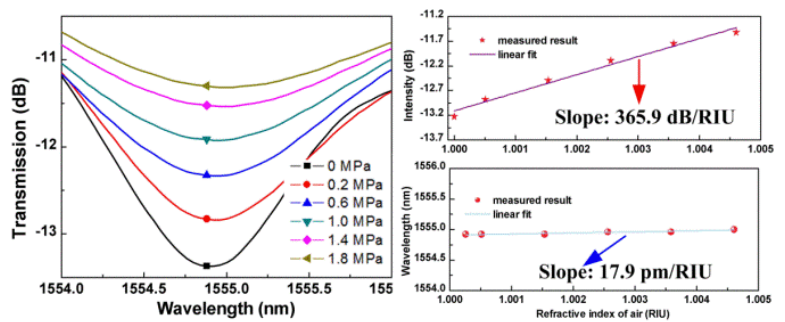

Fig. 3. (a) Spectra response of the sensor for different air pressure. (b) The wavelength shift and dip intensity of the resonance around $1555 \mathrm{~nm}$.

\section{CONCLUSION}

A novel and compact NLG coated HCF sensor was demonstrated by means of splicing a section of HCF to SMFs. The HCF length was chosen as $3 \mathrm{~mm}$ in order to achieve a large fringe contrast of up to $15 \mathrm{~dB}$. Such a sensor based on intensity modulation exhibits a high sensitivity of at the $-365.9 \mathrm{~dB} / \mathrm{RIU}$ at the refractive index of 1 , which is two or three times higher than that of other intensity-modulated RI sensors reported previously. Our RI sensor also exhibits the advantages of compact size ( 3 $\mathrm{mm})$, simple structure, easy fabrication, and good repeatability. However, a complex and large spectrum analyzer has to be employed to measure the change of the dip intensity in our current experiments, which is disadvantageous to RI measurements. So in the practical sensing applications, a simple demodulation device should be developed to measure the intensity at the dip wavelength.

\section{ACKNOWLEDGMENTS}

This work was supported by National Natural Science Foundation of China (61505119, 11174064, 61308027, and 61377090), National Postdoctoral Program for Innovative Talents (BX201600077), Brain Gain
Foundation of Nanjing University of Posts and Telecommunications (NY215040) and the Key Laboratory of Optoelectronic Devices and Systems of Ministry of Education and Guangdong Province (GD201706).

\section{REFERENCES}

[1] C. Caucheteur, T. Guo, F. Liu, B. Guan, J. Albert, "Ultrasensitive plasmonic sensing in air using optical fibre spectral combs," Nature Communications 7, 13371 (2016).

[2] Y. Tan, W. Ji, V. Mamidala, K. Chow, S. Tjin, "Carbon-nanotube-deposited long period fiber grating for continuous refractive index sensor applications," Sens. Actuators B: Chem. 196, 260-264 (2014).

[3] C. Liu, Q. Cai, B. Xu, W. Zhu, L. Zhang, J. Zhao, X. Chen, "Graphene oxide functionalized long period grating for ultrasensitive label-free immunosensing," Biosensors and Bioelectronics 94(15), 200-206 (2017).

[4] Z. Yan, Q. Sun, C. Wang, Z. Sun, C. Mou, K. Zhou, D. Liu, and L. Zhang, "Refractive index and temperature sensitivity characterization of excessively tilted fiber grating," Opt. Express 25(4), 3336-3346 (2017).

[5] Y. Wang, D. Wang, C. Liao, T. Hu, J. Guo, H. Wei, "Temperature-insensitive refractive index sensing by use of micro Fabry-Pérot cavity based on simplified hollow-core photonic crystal fiber," Opt. Lett. 38(3), 269-271, (2013).

[6] Z. Li, Y. Wang, C. Liao, S. Liu, J. Zhou, X. Zhong, Y. liu, K. Yang, Q. Wang, G. Yin, "Temperature-insensitive refractive index sensor based on in-fiber Michelson interferometer," Sens. Actuators B: Chem. 199, 31-35 (2014).

[7] Y. Zhao, F. Xia and J. Li, "Sensitivity-Enhanced Photonic Crystal Fiber Refractive Index Sensor With Two Waist-Broadened Tapers," Journal of Lightwave Technology 34(4), 1373-1379 (2016).

[8] Vanita Bhardwaj, Vinod Kumar Singh, "Fabrication and characterization of cascaded tapered Mach-Zehnder interferometer for refractive index sensing," Sensors and Actuators A: Physical 244, 30-34 (2016).

[9] K. S. Novoselov, A. K. Geim, S. V. Morozov, D. Jiang, Y. Zhang, S. V. Dubonos, I. V.Grigorieva, A. A. Firsov, "Electric field effect in atomically thin carbon films," Science 306, 666-669 (2004).

[10] R. Gao, D. Lu, J. Cheng, Y. Jiang, L. Jiang, Z. Qi, "Humidity sensor based on power leakage at resonance wavelengths of a hollow core fiber coated with reduced graphene oxide," Sensors and Actuators B: Chemical 222, 618-624 (2016).

[11] Y. C. Tan, Z. Q. Tou, K. K. Chow, and C. C. Chan, "Graphene-deposited photonic crystal fibers for continuous refractive index sensing applications," Opt. Express 23, 31286-31294 (2015).

[12] B. C. Yao, Y. Wu, A. Q. Zhang, Y. J. Rao, Z. G. Wang, Y. Cheng, Y. Gong, W. L. Zhang, Y. F. Chen, and K. S. Chiang, "Graphene enhanced evanescent field in microfiber multimode interferometer for highly sensitive gas sensing," Opt. Express 22, 28154-28162 (2014).

[13] Z. L. Ran, Y. J. Rao, W. J. Liu, X. Liao, K. S. Chiang, "Laser-micromachined Fabry-Perot optical fiber tip sensor for high-resolution temperature-independent 
(2) 